\title{
Oxytocin Receptor Gene Methylation: Converging Multilevel Evidence for a Role in Social Anxiety
}

\author{
Christiane Ziegler ${ }^{1,13}$, Udo Dannlowski ${ }^{2,3,13}$, David Bräuer ${ }^{4,13}$, Stephan Stevens ${ }^{5}$, Inga Laeger ${ }^{2,6}$, \\ Hannah Wittmann ${ }^{2}$, Harald Kugel ${ }^{7}$, Christian Dobel ${ }^{8}$, René Hurlemann ${ }^{9}$, Andreas Reif', Klaus-Peter Lesch ${ }^{10}$, \\ Walter Heindel ${ }^{7}$, Clemens Kirschbaum ' ', Volker Arolt ${ }^{2}$, Alexander L Gerlach ${ }^{5}$, Jürgen Hoyer ${ }^{4}$, \\ Jürgen Deckert', Peter Zwanzger 2,12,14 and Katharina Domschke*, ,, 14 \\ 'Department of Psychiatry, University of Würzburg, Würzburg, Germany; ${ }^{2}$ Department of Psychiatry, University of Münster, Münster, Germany; \\ ${ }^{3}$ Department of Psychiatry, University of Marburg, Marburg, Germany; ${ }^{4}$ Institute of Clinical Psychology and Psychotherapy, Technische Universität \\ Dresden, Dresden, Germany; ${ }^{5}$ Institute of Clinical Psychology and Psychotherapy, University of Cologne, Cologne, Germany; ${ }^{6}$ Institute of \\ Psychology, University of Münster, Münster, Germany; ${ }^{7}$ Department of Clinical Radiology, University of Münster, Münster, Germany; ${ }^{8}$ Institute of \\ Biogmagnetism and Biosignal Analysis, University of Münster, Münster, Germany; ${ }^{9}$ Division of Medical Psychology, Department of Psychiatry, \\ University of Bonn, Bonn, Germany; ${ }^{10}$ Division of Molecular Psychiatry, Department of Psychiatry, University of Würzburg, Würzburg, Germany; \\ "Institute of Biopsychology, Technische Universität Dresden, Dresden, Germany; ${ }^{12}$ kbo-Inn-Salzach-Klinikum, Wasserburg am Inn, Germany
}

Social anxiety disorder (SAD) is a commonly occurring and highly disabling disorder. The neuropeptide oxytocin and its receptor (OXTR) have been implicated in social cognition and behavior. This study — for the first time applying a multilevel epigenetic approachinvestigates the role of OXTR gene methylation in categorical, dimensional, and intermediate neuroendocrinological/neural network phenotypes of social anxiety. A total of 110 unmedicated patients with SAD and matched IIO controls were analyzed for OXTR methylation by direct sequencing of sodium bisulfite-converted DNA extracted from whole blood. Furthermore, OXTR methylation was investigated regarding SAD-related traits (Social Phobia Scale (SPS) and Social Interaction Anxiety Scale (SIAS)), salivary cortisol response during the Trier social stress test (TSST), and amygdala responsiveness to social phobia related verbal stimuli using fMRI. Significantly decreased OXTR methylation particularly at CpG Chr3: 8809437 was associated with (I) the categorical phenotype of SAD ( $p<0.00$ I, Cohen's $d=0.535)$, (2) increased SPS and SIAS scores $(p<0.00 \mathrm{I})$, (3) increased cortisol response to the TSST $(p=0.02)$, and $(4)$ increased amygdala responsiveness during social phobia-related word processing (right: $p_{\text {corr }}<0.00 \mathrm{l}$; left: $p_{\text {corr }}=0.005$ ). Assuming that decreased OXTR methylation confers increased OXTR expression, the present finding may reflect a compensatory upregulation for pathologically reduced oxytocin levels or a causally relevant increased OXTR activation in SAD and related traits. OXTR methylation patterns might thus serve as peripheral surrogates of oxytocin tone and aid in establishing accessible biomarkers of SAD risk allowing for indicated preventive interventions and personalized treatment approaches targeting the oxytocin system.

Neuropsychopharmacology (20I5) 40, 1528-1538; doi:I0.1038/npp.20I5.2; published online 28 January 2015

\section{INTRODUCTION}

Social anxiety disorder (SAD) is characterized by marked fear or anxiety about and avoidance of one or more social situations that involve the possibility of being scrutinized, being negatively evaluated by others, or being embarrassed, humiliated, or rejected (American Psychiatric Association, 2013). SAD is a commonly occurring mental disorder affecting 15 million adults, or $6.8 \%$ of the US population,

* Correspondence: Professor K Domschke, Department of Psychiatry, University of Würzburg, Füchsleinstrasse 15, Würzburg D-97080, Germany, Tel: +49 931 20177100, Fax: +49 931 20177109,

E-mail: Domschke_K@ukw.de

${ }^{13}$ Shared first authorship

${ }^{14}$ Shared last authorship

Received 6 September 20 14; revised 21 November 20 I 4; accepted 29

November 20।4; accepted article preview online 7 January 2015 and confers a high individual and socioeconomic burden (Stein and Stein, 2008).

Oxytocin (OT; OXT), a neuropeptide consisting of nine amino acids, is produced in the hypothalamic paraventricular and supraoptic nuclei, is secreted mainly into the posterior lobe of the pituitary gland, and binds to a $G$ protein-coupled receptor (OTR; OXTR) widely expressed in the central nervous system. Oxytocin is frequently termed the 'prosocial neuropeptide' as it facilitates social affiliative and approach behaviors, even in response to social threat, increases positive social interaction, and fosters pair bonding, trust, empathy, and attachment in humans and animals. Furthermore, oxytocin has been suggested as a promising anxiolytic pharmacotherapeutic agent in disorders related to social dysfunctioning (for review see BakermansKranenburg and van Ijzendoorn, 2013; Meyer-Lindenberg et al, 2011). Specifically, in SAD, decreased baseline oxytocin plasma levels have been observed (Hoge et al, 2012), and 
intranasal application of oxytocin as an adjunct to five weekly exposure therapy sessions significantly improved self-reported speech performance compared with placebo (Guastella et al, 2009). On a brain network level, oxytocin attenuates excessive amygdala activation in response to socially relevant or fear-conditioned emotional stimuli (Domes et al, 2007a; Kirsch et al, 2005; Labuschagne et al, 2010; Petrovic et al, 2008)-a brain activation pattern linked to social avoidance and phobia (Phan et al, 2006).

The oxytocin system has furthermore been implicated in social cognition and behavior on a genetic level: the $G$ allele of the $6930 \mathrm{G}>\mathrm{A}$ (rs53576) variant in intron 3 of the oxytocin receptor gene (OXTR) located on chromosome 3 p25.3 was found to be associated with beneficial social traits such as increased empathy, optimism, and trust, whereas A allele carriers displayed lower empathy, lower optimism, and self-esteem, higher levels of state loneliness, more concerns about negative perceptions of company, and higher levels of physiological and dispositional stress reactivity (for review see Kumsta and Heinrichs, 2013).

Recently, epigenetic modifications including methylation of the cytosine pyrimidine ring in $\mathrm{CpG}$ dinucleotides have been suggested as flexible and temporally dynamic regulatory mechanisms that are responsive to environmental stimuli and crucially shape gene expression. The OXTR gene contains a CpG island spanning exons 1 to 3 (Chr3:8 808 962-8 811 280, GRCh37/hg19) (Kumsta et al, 2013), with evidence for increased OXTR methylation to confer decreased OXTR expression: in hepatoblastoma cells, methylation of this OXTR CpG island significantly suppressed OXTR mRNA expression by $70 \%$ (Kusui et al, 2001). Accordingly, Gregory et al (2009) observed methylation of two CpG sites within this island to result in a $20 \%$ reduction in OXTR mRNA expression, and lower plasma oxytocin levels were associated with increased OXTR methylation in peripheral blood (Dadds et al, 2014). Also, in murine cell lines, negative correlations between OXTR methylation and mRNA expression were observed (Mamrut et al, 2013). So far, OXTR methylation of this CpG island has been investigated with regard to several phenotypes related to social cognition and functioning: in a sample of male adolescents with oppositional-defiant or conduct disorder, increased OXTR methylation in peripheral blood was associated with callous-unemotional traits (Dadds et al, 2014). In autism, significantly increased OXTR methylation was observed in peripheral blood mononuclear cells and temporal cortex tissue (Gregory et al, 2009). Applying an imaging epigenetic approach in healthy subjects, Jack et al (2012) discerned increased OXTR methylation to be associated with neural processes underlying social interpretation of ambiguous stimuli, particularly enhanced activation in the temporoparietal junction and the dorsal anterior cingulate cortex. Finally, healthy subjects displayed increased peripheral blood OXTR methylation in the proteincoding part of exon 3 directly after exposure to the Trier social stress test (TSST), but significantly decreased methylation at follow-up (Unternaehrer et al, 2012).

Given the above-mentioned findings of decreased baseline oxytocin plasma levels in SAD and, reciprocally, of oxytocin improving clinical symptoms in patients with SAD and attenuating neural correlates of social anxiety, it is hypothesized that one key pathomechanism of SAD might be a diminished oxytocin synthesis and/or OXTR dysregulation possibly linked to epigenetic mechanisms. Thus, in this study we for the first time applied a multilevel epigenetic approach to elucidate the role of OXTR methylation in categorical, dimensional, and intermediate neuroendocrinological/neural network phenotypes of social anxiety. In detail, it was investigated whether OXTR methylation was associated (1) with the categorical phenotype of SAD, (2) with dimensional traits related to social anxiety in both patients and healthy probands, (3) with cortisol response to the TSST as a nonclinical intermediate phenotype of social anxiety in healthy probands, and (4) with brain activation correlates of social phobia-related word processing in patients with SAD.

\section{PATIENTS AND METHODS}

\section{Samples and Procedures}

Overall sample. The overall sample comprised 111 SAD patients of Caucasian descent recruited at the Department of Psychiatry and the Institute of Psychology, University of Muenster, Germany $(\mathrm{f}=77, \mathrm{~m}=34$; age $=30.1+9.9$ years $+\mathrm{SD})$. The diagnosis of $\mathrm{SAD}$ was ascertained by experienced psychiatrists and/or clinical psychologists on the basis of medical records and structured clinical interviews (SCID-I) according to the criteria of DSM-IV (Wittchen, 1997). A total of 21 patients were comorbidly diagnosed with a concurrent mild to moderate major depressive episode (18.9\%; data lacking for 7 patients), in line with epidemiological data reporting $19.5-35 \%$ of individuals with primary SAD meeting diagnostic criteria for currently comorbid or lifetime major depression, respectively (Ohayon and Schatzberg, 2010; Stein et al, 1990), 5 patients with specific phobia, and 1 patient with panic disorder and agoraphobia. Patients with psychotic disorders including schizoaffective disorder, bipolar disorder, mental retardation, or neurological/neurodegenerative disorders impairing psychiatric evaluation were not included in this analysis. Current or past drug or alcohol abuse or dependence and any severe somatic disorder were further exclusion criteria. Axis II disorders were not evaluated and thus not explicitly excluded. Females were not included if pregnant. Some of the patients were included in previous studies on a different topic (Domschke et al, 2009; Stevens et al, 2008). Patients (except one male patient on $15 \mathrm{mg}$ citalopram every other day) were not taking any psychoactive medication so that a confounding effect of antidepressant medication such as serotonin reuptake inhibitors (SSRIs), which have been suggested to elevate oxytocin levels (Uvnas-Moberg et al, 1999), can be excluded.

The control group comprised 111 healthy Caucasian subjects matched to the patient group by sex $(\mathrm{f}=77$, $\mathrm{m}=34 ; p=0.56)$ and age $(30.9+10.5$ years $+\mathrm{SD} ; p=0.59)$. Absence of DSM-IV axis I disorders was established by experienced psychologists on the basis of a structured clinical interview (Mini International Neuropsychiatric Interview (MINI); SCID-I) according to the criteria of DSM-IV (Wittchen, 1997). Current or past drug or alcohol abuse or dependence and any severe somatic disorder were exclusion criteria. Females were excluded if pregnant. 
Table I Characteristics of Patient and Control Sample

\begin{tabular}{lcc}
\hline Characteristics & $\begin{array}{c}\text { Patients } \\
(\mathbf{N}=\mathbf{I} \mathbf{~ I 0})\end{array}$ & $\begin{array}{c}\text { Controls } \\
(\mathbf{N}=\mathbf{I} \mathbf{1 0})\end{array}$ \\
\hline Sex & $\mathrm{f}=76 ; \mathrm{m}=34$ & $\mathrm{f}=77 ; \mathrm{m}=33$ \\
Age (yrs; mean + SD) & $30.1+9.9$ & $30.9+10.5$ \\
OXTR rs53576 genotype & $\mathrm{AA}=9 ; \mathrm{AG}=53 ;$ & $\mathrm{AA}=12 ; \mathrm{AG}=48 ;$ \\
& $\mathrm{GG}=48$ & $\mathrm{GG}=50$ \\
OXTR rs53576 grouped & 62 vs 48 & $60 \mathrm{vs} 50$ \\
genotype (AA/AG vs GG) & & $3.6 \pm 0.4(\mathrm{~N}=108)$ \\
SPS scores (mean $\pm \mathrm{SE})$ & $36.6 \pm 1.3(\mathrm{~N}=110)$ & $9.1 \pm 0.6(\mathrm{~N}=108)$ \\
SIAS scores (mean $+\mathrm{SE})$ & $45.5 \pm 1.3(\mathrm{~N}=110)$ &
\end{tabular}

Abbreviations: f, female; $m$, male; $N$, sample size; SPS, Social Phobia Scale (Stangier et al, 1999); SIAS, Social Interaction Anxiety Scale (Stangier et al, 1999).

Patient and control samples did not differ regarding $\operatorname{sex}\left(\chi^{2}=0.02, p=0.88\right)$, age (Mann-Whitney $U$-test, $p=0.46)$, OXTR rs53576 genotype $\left(\chi^{2}=0.72\right.$, $p=0.70)$, or OXTR rs53576 grouped genotype AA/AG vs GG $\left(\chi^{2}=0.07\right.$, $p=0.79$ ). SPS and SIAS scores differed significantly between the two groups (Mann-Whitney U-test, $p<0.00 \mathrm{I}$ ).

Dimensional measures of social anxiety severity were assessed in patients $(N=110)$ and controls $(N=108)$ using the following psychometric instruments: Social Phobia Scale (SPS; Stangier et al, 1999) and Social Interaction Anxiety Scale (SIAS; Stangier et al, 1999) (Table 1).

fMRI sample. A total of 25 female patients (age $=28.8+8.3$ years $+\mathrm{SD}$ ) with current SAD according to DSM-IV criteria (SCID-I) constituting a subsample of the overall sample of SAD patients described above also took part in an fMRI experiment assessing amygdala responsiveness to social phobia-relevant verbal stimuli in relation to OXTR methylation status. This patient subsample and the fMRI task have been described elsewhere (Laeger et al, 2014a), with patients showing greater amygdala responsiveness to social phobiarelated words than to generally negative words when compared with healthy controls. All patients fulfilled the inclusion/exclusion criteria mentioned above for the overall sample and did not take any psychotropic medication including benzodiazepines. Comorbid diagnoses in the fMRI sample were currently remitted depressive disorder $(N=3)$, currently remitted single major depressive episode $(N=2)$, currently remitted anorexia nervosa $(N=1)$, and specific phobia $(N=3)$. None of them had a comorbid diagnosis of a current major depressive episode or generalized anxiety disorder. Additional exclusion criteria for all fMRI patients were neurological illnesses or a history of seizures or head trauma, head movements of $>2 \mathrm{~mm}$ and/or $2^{\circ}$, and the general MRI contraindications. All participants were German native speakers and had normal or corrected-to-normal vision.

TSST sample. An independent sample of $N=20$ healthy subjects $(\mathrm{f}=9, \mathrm{~m}=11$; age $=26.7+9.0$ years $+\mathrm{SD})$ was recruited via advertisement at the Institute of Psychology, University of Dresden, Germany, for analysis of a potential association of OXTR methylation and salivary cortisol response to a standardized stress situation (TSST; Kirschbaum et al, 1993). Subjects were included if the stem questions of the Composite International Diagnostic Interview (DIA-X/M-CIDI (Wittchen and Pfister, 1997) indicated no lifetime psychiatric disorder, and if the individuals did not smoke $>10$ cigarettes per day (Kirschbaum et al, 1992), and had not participated in a study applying the TSST before.

This study was approved by the ethics committees of the Universities of Muenster and Dresden, Germany, and was conducted according to the ethical principles of the Helsinki Declaration. Written informed consent was obtained from all participants.

\section{Isolation of DNA}

DNA was isolated from human whole blood using the FlexiGene DNA Kit (QIAGEN, Hilden, Germany) according to the manufacturer's instructions.

\section{Genotyping of OXTR rs53576}

Isolated DNA was genotyped for the OXTR SNP rs53576 according to published protocols (Wu et al, 2005) with minor modifications. DNA was amplified by PCR ( $45 \mathrm{~s}$ at $95^{\circ} \mathrm{C}, 45 \mathrm{~s}$ at $62.5^{\circ} \mathrm{C}, 45 \mathrm{~s}$ at $72{ }^{\circ} \mathrm{C}$ for 35 cycles) using oligonucleotide primers F: $5^{\prime}$-ATTTGTACCCAGAAGGGC CG- ${ }^{\prime}$ and R: $5^{\prime}$-ACAGAACTGGCAACCTGGAA- ${ }^{\prime}$ flanking the polymorphic region. PCR products were run on a $3 \%$ agarose gel and visualized by ethidium bromide staining using ultraviolet light. Genotypes were determined by investigators blinded for clinical diagnoses. Hardy-Weinberg criteria, as calculated by the online program DeFinetti (http://ihg.gsf.de/cgi-bin/hw/hwa1.pl; TF Wienker and TM Strom), were fulfilled for OXTR rs53576 genotype distribution (patients: $\mathrm{AA}=9, \mathrm{AG}=53, \mathrm{GG}=49, p=0.39$; controls: $\mathrm{AA}=12, \mathrm{AG}=49, \mathrm{GG}=50, p=1.00)$. For further analyses, genotypes were grouped according to previous studies (AA/ AG vs GG; see, eg, Rodrigues et al, 2009; Saphire-Bernstein et al, 2011).

\section{Bisulfite Sequencing}

Treatment of DNA with sodium bisulfite converts nonmethylated cytosine to uracil, whereas methylated cytosine remains unaltered. Aliquots of isolated DNA were treated with sodium bisulfite using the EZ DNA Methylation-Gold Kit (Zymo Research, HiSS Diagnostics GmbH, Freiburg, Germany) according to the manufacturer's protocol for all samples in one batch. Briefly, $450 \mathrm{ng}$ of DNA was submitted to bisulfite treatment at $98^{\circ} \mathrm{C}$ for $10 \mathrm{~min}$ and $53^{\circ} \mathrm{C}$ for $4 \mathrm{~h}$ in a thermal cycler (Professional Thermocycler, Biometra). DNA was eluted after desulfonation and purified using Zymo-Spin IC columns (Zymo Research, HiSS Diagnostics $\mathrm{GmbH}$, Freiburg, Germany). As a control, commercially available fully methylated and fully nonmethylated DNA (Human Methylated and Non-Methylated DNA Set, Zymo Research, HiSS Diagnostics $\mathrm{GmbH}$ ) was used in all experiments.

An amplicon covering exon 3 of the OXTR gene was chosen as the target sequence for further analysis in analogy to a previous study on OXTR gene methylation investigating 
a

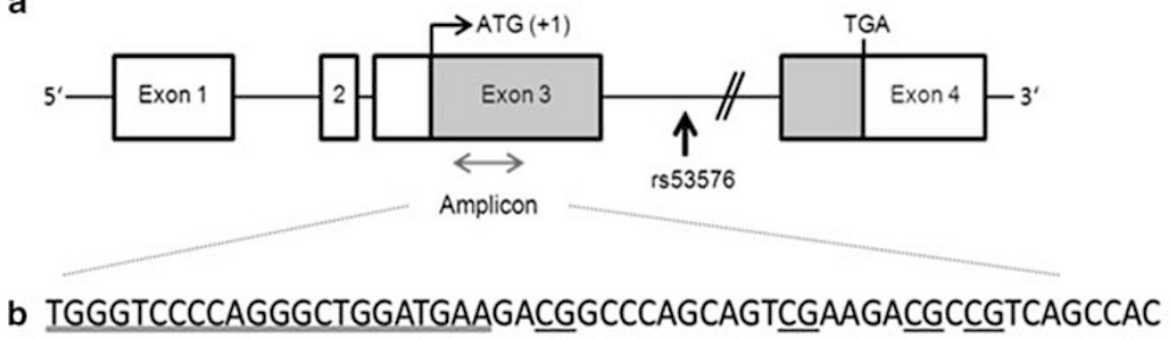

CTCGCGCAGAGAGAAGATGTGCACCTGCGGCGCGTGGCCACCAGGCAGCCGAGC

CACGTGGCGAGCACTGCCAGGCGGTCG GTGCGGCGGCGCAGCGAGCGCAGCGGC

TGGCAGATGGCCAGGCAGCGGTCCAGGGACATGAGCAGCAGCAGGTAGGTGGAG

\section{GCGAACATGCCCACCACCTGCAAGTACTTGACCAG}

Figure I (a) The OXTR gene on chromosome 3p25-3p26.2 consists of four exons with the translation start site $($ ATG $(+1))$ in exon 3 and the stop codon (TGA) in exon 4. OXTR SNP rs53576 is localized in the third intronic region (not to scale). The presently analyzed amplicon (not to scale) is located within OXTR exon 3 downstream to the translation start site (Kumsta et al, 2013; Mamrut et al, 20l3; Unternaehrer et al, 20l2). (b) The sequence is displayed as per GRCh37 build, National Center for Biotechnology Information (NCBI) reference sequence NC_0000 I7.I0 (Chr3:8 809 28I-8 809 534), and encompasses the presently analyzed amplicon with primer pair binding sites double underlined. CpG sites $I-12$ analyzed in the present study are boxed and numbered $(\mathrm{CpGl}=$ Unternaehrer $(U)$ CpG2 (Unternaehrer et al, 20I 2) = Chr3: 8809464 (NC_0000 I 7. I0), CpG2=(U) CpG3=Chr3: 8809442 ,

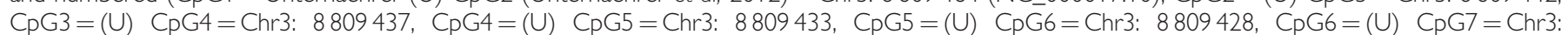

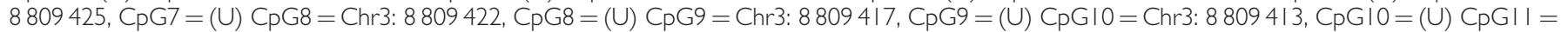
Chr3: 8809 399, CpGII = (U) CpGI2= Chr3: 8809 394, CpGI2=(U) CpGI3=Chr3: 8009 387), CpGs contained in the amplicon, but not readable because of technical difficulties particularly at the $3^{\prime}$ and $5^{\prime}$ ends of the amplicon, are underlined.

a phenotype related to social cognition and functioning (Unternaehrer et al, 2012). This amplicon spans the region Chr3: 8809 281-8 809534 (UCSC Human Genome Browser; February 2009; GRCh37/hg19) and contains 22 CpG sites. The amplicon was PCR-amplified using oligonucleotide primers designed for bisulfate-converted DNA (5'-TTGG TTAAGTATTTGTAGGTGGTG- ${ }^{\prime}$ and $5^{\prime}$-TAAATCCCCAA AACTAAATAAAAAC- $3^{\prime}$, length: $253 \mathrm{bp}$ ) under the following PCR conditions using the HotStarTaq Plus Master Mix Kit (QIAGEN): denaturation at $94{ }^{\circ} \mathrm{C}$ for $1 \mathrm{~min}$, annealing at $59.5^{\circ} \mathrm{C}$ for $1 \mathrm{~min}$, and extension at $72^{\circ} \mathrm{C}$ for $2 \mathrm{~min}(40$ cycles).

Amplified PCR products were purified (EXO/SAP (Exonuclease I/Shrimp Alkaline Phosphatase) clean-up) and sequenced by LCG Genomics (Berlin, Germany). The sequencing services were delivered on the ABI $3730 \mathrm{XL}$ platforms for traditional Sanger sequencing. Because of technical difficulties particularly at the $3^{\prime}$ and $5^{\prime}$ ends of the amplicon, in the present sample, electropherograms were robustly readable for $12 \mathrm{CpG}$ sites using Chromas (Technelysium) and Sequence Scanner software (Applied Biosystems by Life Technologies, Darmstadt, Germany). These $12 \mathrm{CpG}$ sites were therefore included in all further analyses (Figure 1).

The obtained sequences were quantitatively analyzed by determining relative peak heights $(\mathrm{C} / \mathrm{C}+\mathrm{T})$ from the normalized sequence trace files using the Epigenetic Sequencing Methylation analysis software (ESME) specifically designed and evaluated for artificially generated SNPs (C/T and G/A). ESME performs quality control, normalizes signals, corrects for incomplete bisulfite conversion, and aligns generated bisulfite sequence and reference sequence to compare $\mathrm{C}$ with $\mathrm{T}$ peak heights at $\mathrm{CpG}$ sites (Lewin et al, 2004) as successfully used to analyze methylation profiles in other psychiatric phenotypes (see, eg, Alasaari et al, 2012; Domschke et al, 2013; Domschke et al, 2012; Domschke et al, 2014b; Domschke et al, 2014a; Tadic et al, 2013). To account for run variability, all samples were tested in duplicate, yielding a mean individual methylation score for each $\mathrm{CpG}$.

\section{Statistical Analysis}

In the overall sample, categorical data were analyzed using the $\chi^{2}$ test. Differences in continuous variables were evaluated by Student's $t$-test and multivariate ANOVA corrected for age and genotype, respectively. Correlations between dimensional variables (eg, SPS and SIAS) and methylation were calculated by means of correlation or regression analyses. The post hoc Bonferroni correction for multiple comparisons regarding the main outcome variable (OXTR methylation in patients $v s$ controls) set the significance level to $p \leq 0.004$ ( $p=0.05$ divided by 13 tests, as methylation across the entire amplicon plus methylation at 12 individual $\mathrm{CpGs}$ were tested). The overall sample size of $N=220$, with an assumed $\alpha$-error of 0.05 and a low effect 
size of 0.10 when applying MANOVA (2 groups, 13 response variables), achieved a post hoc power of $87 \%$ ( $\mathrm{G}^{\star}$ Power, Version 3.1.9.2; Faul et al, 2007).

\section{Trier Social Stress Test}

All female participants were tested in the luteal phase of their menstrual cycle (Kirschbaum et al, 1999). Participants were requested to abstain from alcohol the evening before and on the day of testing, they were asked to have a regular meal on the testing day, and to avoid smoking, drinking, and eating $2 \mathrm{~h}$ before arriving at the laboratory. The stress paradigm comprised three phases: a 60 min rest period, a $15 \mathrm{~min}$ stressor period (TSST), and a $60 \mathrm{~min}$ post-stressor period. The TSST (Kirschbaum et al, 1993) consisted a short preparation period $(5 \mathrm{~min})$, a standardized public speaking task ( $5 \mathrm{~min})$, and a mental arithmetic task $(5 \mathrm{~min})$ in front of a committee. Saliva samples were obtained $45(\mathrm{t} 1)$ and $1 \mathrm{~min}(\mathrm{t} 2)$ before, and 1 (t3), 10 (t4), 20 (t5), 30 (t6), 45 (t7), and 60 (t8) min after the TSST. For testing the maximum salivary cortisol response to the TSST, the highest salivary cortisol level at time points $\mathrm{t} 3$ to $\mathrm{t} 5$ was selected, as peak levels of cortisol are to be expected with a latency of 10$20 \mathrm{~min}$ after a presented stressor (Kudielka et al, 2007). Furthermore, we calculated a delta score subtracting baseline salivary cortisol ( $\mathrm{t} 2$ ) from the maximum salivary cortisol level at time points $t 3$ to $t 5$. Four individuals with a cortisol increase of $<1.5 \mathrm{nmol} / 1$ were defined as nonresponders (Miller et al, 2013) and therefore excluded for all further analyses. To analyze the potential association of OXTR mean methylation across all $12 \mathrm{CpG}$ sites and specifically at $\mathrm{CpG} 3$, respectively, with maxima and delta measures of salivary cortisol, pairwise partial correlations with age as covariate were calculated.

\section{fMRI Task}

Stimulus material consisted of a total of 96 German nouns, 24 negative (eg, pain, victim), social phobia-related (eg, disgrace, audience), positive (eg, holidays, baby), and neutral (eg, pencil, arm) words. Negative, positive, and neutral words constituted a subset of the stimuli from Kissler et al (2007) that have already been shown to induce amygdala activation in healthy subjects (Laeger et al, 2012). Social phobia-related nouns were taken from Schmidt et al (2010), who demonstrated appropriate limbic and frontal activations in response to these stimuli in 19 patients with SAD. All word categories were matched for word length and frequency of use (CELEX database; Baayen et al, 1995). Words were presented in white color in the center of a black screen in alternating $15 \mathrm{~s}$ blocks of 12 words per block. Presentation time was $1000 \mathrm{~ms}$ per word, with a fixed interstimulus interval of $250 \mathrm{~ms}$. The blocks of words were presented in a pseudorandomized order. A $10 \mathrm{~s}$ resting phase (white fixation cross in the center of a black screen) was following each block of words. In all, the paradigm took $400 \mathrm{~s}$ (6:40 min), and consisted of 8 word blocks (2 negative, 2 social phobia related, 2 positive, and 2 neutral). The stimuli were projected onto a screen at the rear end of the MR tunnel, using a projector shielded against radio frequency interference. Each block was presented two times and the participants were instructed to read the words attentively.

\section{fMRI Methods}

T2* functional data were acquired on a 3-Tesla scanner (Gyroscan Intera T3.0, Philips Medical Systems, Best, The Netherlands) using a single-shot echo planar imaging (EPI) sequence (whole brain coverage, $\mathrm{TE}=30 \mathrm{~ms}, \mathrm{TR}=2.5 \mathrm{~s}$, $\mathrm{FA}=90^{\circ}, 40$ slices, slice thickness $3.6 \mathrm{~mm}$, no gap, matrix $64 \times 64$, FOV $230 \mathrm{~mm}$, in-plane resolution $3.6 \mathrm{~mm} \times 3.6 \mathrm{~mm}$ ). The slices were tilted $25^{\circ}$ from the anterior commissure/ posterior commissure line to minimize dropout artifacts in the orbitofrontal and mediotemporal regions. Functional imaging data were analyzed using Statistical Parametric Mapping (SPM8, http://www.fil.ion.ucl.ac.uk/spm) using published protocols (see, eg, Laeger et al, 2014b). Images were realigned and unwarped, spatially normalized to standard MNI space (Montreal Neurological Institute), and smoothed with a Gaussian kernel of $6 \mathrm{~mm}$ full width at half maximum (FWHM). Onsets and durations of the different word conditions were modeled with a canonical hemodynamic response function based on the general linear model. As we focused on amygdala responsiveness to social phobia-related words (in contrast to neutral or generally negative words), two individual fixed-effects first-level contrasts (social phobia-related $>$ neutral words, and social phobia-related $>$ negative words) were calculated for each subject, and the resulting contrast images were entered in the second-level (group) random-effects analysis. A multiple regression model predicting amygdala responsiveness by OXTR methylation status was conducted. Age was entered as nuisance regressor. Based on the results in the overall sample, we used mean methylation across all $12 \mathrm{CpG}$ sites as well as methylation rate specifically at $\mathrm{CpG} 3$ as predictors. According to our hypothesis, a region of interest (ROI) analysis of the bilateral amygdala (defined according to the AAL Atlas; Tzourio-Mazoyer et al, 2002) was performed. In order to control for multiple statistical testing, group results were calculated with a combined height and extent threshold based on Monte Carlo simulations, as implemented in the AlphaSim procedure (Forman et al, 1995). A corrected false-positive detection rate at $p<0.05$ was maintained, with a cluster extent $(k)$ empirically determined by computing 5000 simulations (yielding $k=38$ for the bilateral amygdala).

\section{RESULTS}

\section{Sample Characteristics}

A total of 111 patients with social anxiety disorder and 111 controls were matched for sex and age. Because of nonsufficient quality of sequencing data for methylation analysis (see Patients and methods), sample size was reduced to $N=220(\mathrm{f}=153, \mathrm{~m}=67)$ with complete methylation status at all $12 \mathrm{CpG}$ sites being available for $N=110$ patients (f=76, $\mathrm{m}=34)$ and $N=110$ healthy subjects $(\mathrm{f}=77$, $\mathrm{m}=33)$. Characteristics of the patient and control samples entering further analyses regarding age, sex, OXTR rs53576 genotype status, SPS, and SIAS scores are given in Table 1. 


\section{OXTR Variation and SAD}

OXTR rs53576 grouped genotype distribution (AA/AG vs GG) did not differ significantly between SAD patients and healthy controls (Table $1 ; \chi^{2}=0.074, p=0.786$ ).

\section{OXTR DNA Methylation Status}

In the combined sample across patients and controls, only minor average methylation across all $12 \mathrm{CpGs}$ was discerned in the investigated amplicon (mean + SD: $0.157 \pm 0.106$, individual min: 0.000, individual max: 0.440), with levels of methylation at single $\mathrm{CpGs}$ varying considerably with a range (individual min-max) between 0.000 and 0.685 (CpG1: mean + SD: $0.132 \pm 0.106, \quad$ CpG2: $0.078+0.077, \quad$ CpG3: $0.228+0.174$, CpG4: $0.120+0.108$, CpG5: $0.212+0.159$, CpG6: $0.218+0.151$, CpG7: $0.185+0.134$, CpG8: $0.118+$ 0.097, CpG9: $0.182+0.134$, CpG10: $0.214 \pm 0.153$, CpG11: $0.067 \pm 0.065$, and CpG12: $0.133 \pm 0.105)$. Correlations between individual methylation levels at all $12 \mathrm{CpG}$ residues calculated by pairwise correlations ranged between 0.53 and 0.95 reaching statistical significance $(p<0.05)$ for correlation between all $12 \mathrm{CpG}$ sites.

\section{Influence of Age, Sex, Depression, and OXTR rs53576 Genotype on OXTR Methylation}

In the overall sample, age was positively correlated with average methylation status across all $12 \mathrm{CpGs}$ as well as at individual CpG sites 2 and 5-12 $(r=0.14-0.22, p<0.05)$. Therefore, age was included as a covariate in further analyses. Neither overall OXTR methylation nor methylation at single CpGs in the analyzed amplicon showed association with sex $(p>0.26)$ or comorbid major depression $(p>0.24)$. Average OXTR DNA methylation $(p=0.007)$ and methylation at CpG sites $1,2,5-10$, and $12(p=0.03-$ 0.001) were associated with OXTR rs53576 genotype, in that A allele carriers showed decreased methylation. When analyzing patients and controls separately, this latter association seemed to be driven by the patient group (patients: average methylation as well as methylation at $\mathrm{CpG}$ sites $1-12 ; p=0.05-0.003$; controls: average methylation as well as methylation at $\mathrm{CpG}$ sites $1-12 ; p=0.10-0.95$; for details see Supplementary Table 1).

\section{OXTR Methylation and SAD}

Average methylation across all 12 OXTR CpG sites was significantly lower in patients with $\mathrm{SAD}$ as compared with healthy controls (patients: mean + SD $0.151+0.114$, controls: $0.164+0.097 ; p=0.03$; Cohen's $d=0.119)$. Multivariate analysis of variance for single CpG sites corrected for age revealed a significant effect of group $(p<0.001)$, with univariate tests showing significantly lower methylation in patients than in healthy controls at $\mathrm{CpG}$ sites $2,3,6,7,8$, and 9, with $p$-values ranging from 0.03 to $<0.001$ (Table 2). Only at CpG12, methylation was significantly higher in patients than in controls (Table 2). After Bonferroni correction for multiple testing (corrected $p$-value of $<0.004$; see Patients and methods) that-given strong correlations between individual methylation levels at all $12 \mathrm{CpG}$ residues (see above)-is extremely conservative, association of
Table 2 Mean Methylation at Single OXTR CpG Sites in SAD Patients and Controls

\begin{tabular}{|c|c|c|c|c|c|c|}
\hline \multirow[t]{2}{*}{ Position } & \multicolumn{2}{|c|}{$\begin{array}{l}\text { Patients } \\
(N=I \mid 0)\end{array}$} & \multicolumn{2}{|c|}{$\begin{array}{l}\text { Controls } \\
(N=I 10)\end{array}$} & \multirow[t]{2}{*}{$P$-value } & \multirow[t]{2}{*}{ Cohen's d } \\
\hline & Mean & SE & Mean & SE & & \\
\hline CpGI & 0.141 & 0.010 & 0.123 & 0.010 & 0.29 & -0.173 \\
\hline CpG2 & 0.078 & 0.007 & 0.079 & 0.008 & $0.03 *$ & 0.012 \\
\hline CpG3 & 0.183 & 0.014 & 0.273 & 0.018 & $<\left.0.00\right|^{*} * * * *$ & 0.535 \\
\hline CpG4 & 0.109 & 0.009 & 0.131 & 0.011 & 0.07 & 0.120 \\
\hline CpG5 & 0.202 & 0.015 & 0.223 & 0.015 & 0.06 & 0.136 \\
\hline CpG6 & 0.204 & 0.015 & 0.232 & 0.014 & $0.02 *$ & 0.184 \\
\hline CpG7 & 0.179 & 0.014 & 0.192 & 0.012 & $0.03 *$ & 0.096 \\
\hline CpG8 & 0.116 & 0.009 & 0.120 & 0.009 & $0.01 * *$ & 0.043 \\
\hline CpG9 & 0.178 & 0.013 & 0.186 & 0.012 & $0.01 * *$ & 0.061 \\
\hline CpGIO & 0.213 & 0.016 & 0.214 & 0.013 & 0.10 & 0.006 \\
\hline CpGII & $0.07 \mid$ & 0.006 & 0.064 & 0.006 & 0.07 & -0.108 \\
\hline CpGI2 & 0.139 & 0.011 & 0.128 & 0.009 & $0.01 * *$ & -0.109 \\
\hline
\end{tabular}

Mean methylation levels at single OXTR CpG sites for patients with social anxiety disorder (SAD) and healthy controls (mean; standard error (SE)). $N=$ sample size, $p$-value from MANOVA corrected for age.

*significant at $p \leq 0.05$; **significant at $p \leq 0.01$; ***significant at $p \leq 0.00$ l.

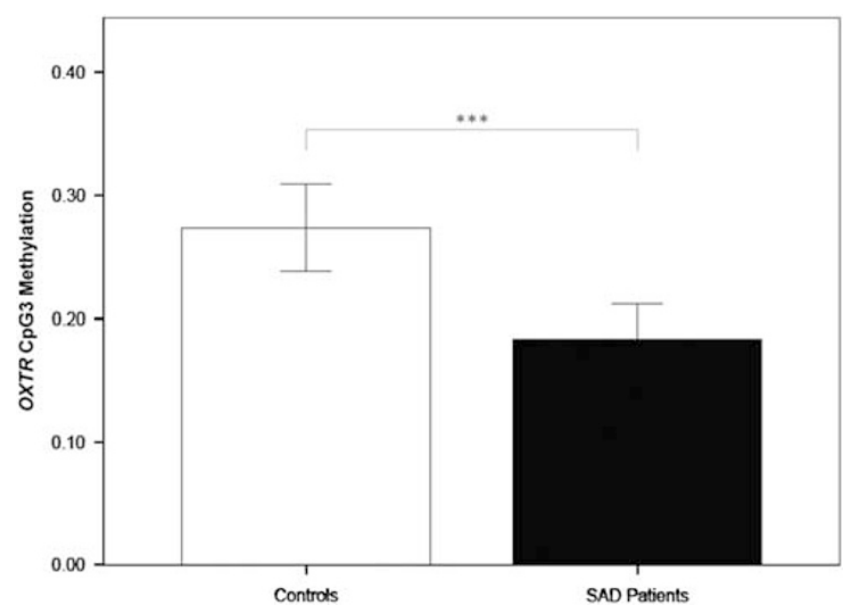

Figure 2 Mean methylation at OXTR CpG3 (Chr3: 8 809 437) in healthy controls $(N=110$; white bar, mean $=0.273)$ and patients with social anxiety disorder (SAD; $N=1 \mid$ o; black bar, mean $=0.183)$. Error bar $\pm S E$. *****ignificant at $p<0.001$.

hypomethylation at CpG3 (Chr3:8 809437) with SAD remained significant (Figure 2).

When additionally considering OXTR rs53576 genotype (AG/AG vs GG) as second fixed factor in the MANCOVA (see above) in order to control for an influence of genotype on methylation, results remained stable $(p<0.001)$, with univariate tests showing significantly lower methylation in patients than in healthy controls across all 12 OXTR CpG sites $(p=0.02)$ and at individual $\mathrm{CpG}$ sites $2,3,6,7,8,9$, and $12(p=0.05-0.001)$. 


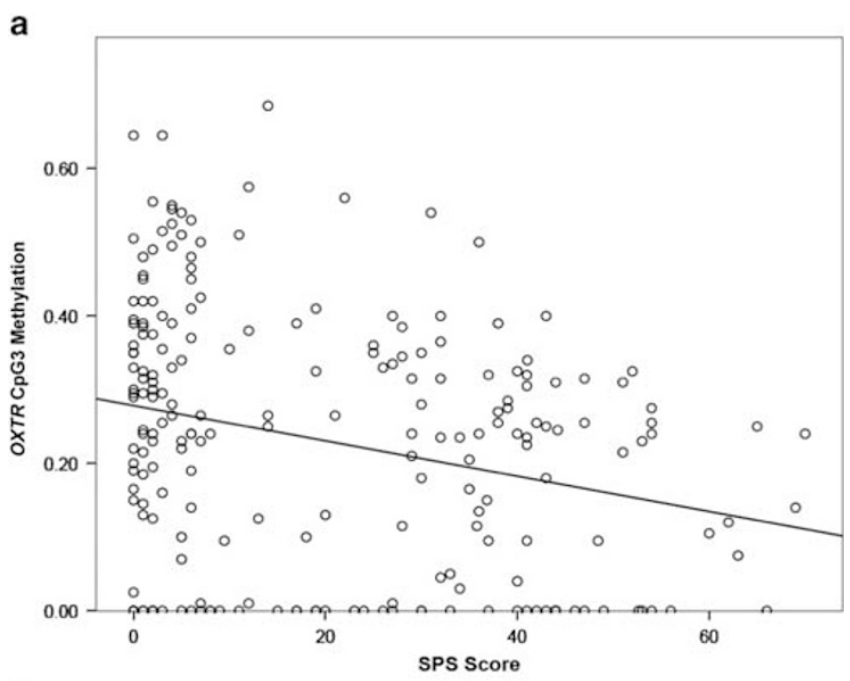

b

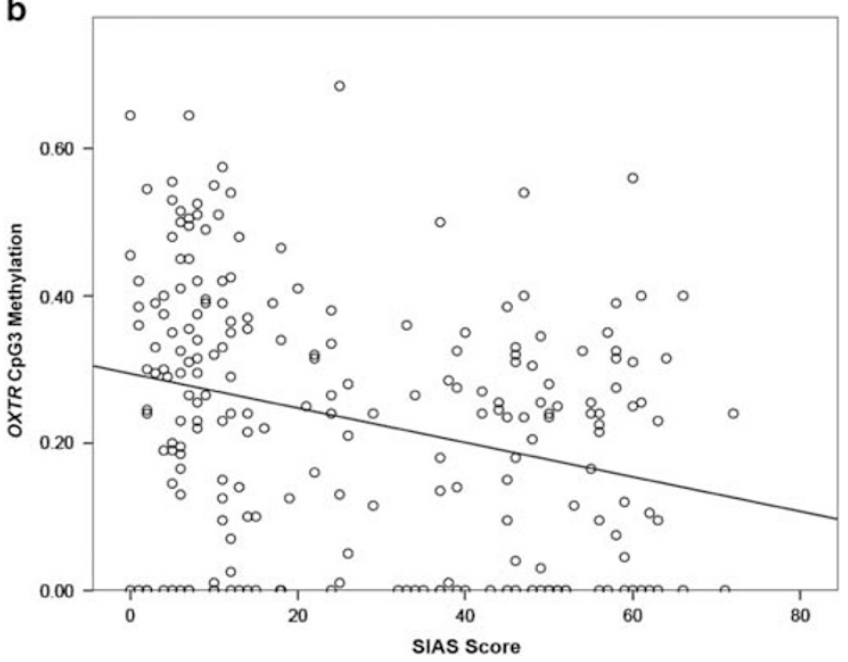

Figure 3 (a) Correlation of decreased methylation at OXTR CpG3 (Chr3: 8809 437) with increased Social Phobia Scale (SPS; Stangier et al, 1999) scores $(r=-0.39, p<0.001)$ and (b) Social Interaction Anxiety Scale (SIAS; Stangier et al, 1999) scores $(r=-0.40, p<0.00 \mathrm{I})$ in the combined sample of II0 patients with social anxiety disorder and 108 healthy controls (data missing for two controls).

\section{OXTR Methylation and Dimensional Traits Related to Social Anxiety}

In the combined sample $(N=218$; patients $=110$, controls $=$ 108), a negative correlation between average OXTR methylation across all 12 CpGs and scores on the SPS (Stangier et al, 1999); $r=-0.20, p=0.02$ ) and the SIAS (Stangier et al, 1999); $r=-0.18, p=0.04$ ) was detected. This was particularly robust for OXTR CpG3, with decreased methylation at CpG3 correlating significantly with increased SPS $(r=-0.39, p<0.001$; Figure $3 \mathrm{a}$ ) and SIAS $(r=-0.40, p<0.001$; Figure $3 \mathrm{~b})$ scores.

\section{OXTR Methylation and Cortisol Response to the TSST}

In healthy subjects $(N=16)$, a significant negative correlation of OXTR methylation and maximum salivary cortisol response to the TSST was observed for average OXTR methylation across all 12 CpGs $(r=-0.56, p=0.03$; Figure 4) and $\mathrm{CpG} 3$ in particular $(r=-0.59, p=0.02)$.

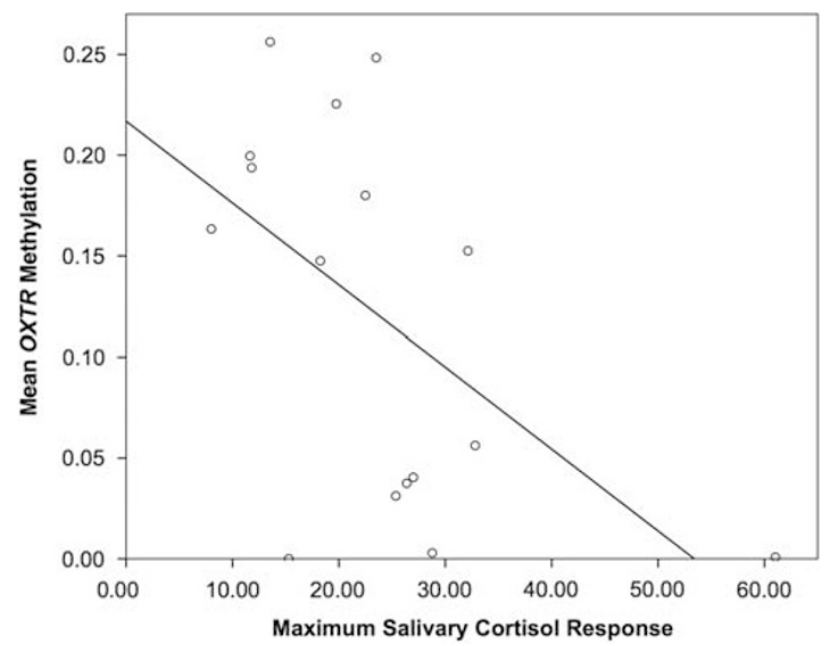

Figure 4 Correlation of decreased mean OXTR methylation across all 12 CPGs with increased cortisol salivary cortisol response after the Trier social stress test (TSST) in healthy subjects $(N=16)(r=-0.56, p=0.03)$.

The same pattern was discerned for association of delta salivary cortisol response to the TSST and OXTR methylation, both across all $12 \mathrm{CpGs}(r=-0.56, p=0.03)$ and CpG3 $(r=-0.53, p=0.04)$.

\section{OXTR Methylation and Neural Responsiveness During Social Phobia-Related Word Processing}

In patients with $\operatorname{SAD}(N=25)$, a strong negative correlation of mean OXTR methylation across all $12 \mathrm{CpGs}$ and amygdala responsiveness to social phobia-related words $v s$ generally negative words was discerned (right: $x=26$, $y=-2, \quad z=-14, \quad Z=3.70, \quad k=178, \quad p_{\text {corr }}=0.0002$; left: $x=-30, y=0, z=-28, Z=2.68, k=118, p_{\text {corr }}=0.0018$; Figure 5). Similar values were found for methylation at CpG3 (right: $x=28, y=-2, z=-12, Z=3.26, k=172$, $p_{\text {corr }}=0.0002$; left: $x=-24, y=0, \quad z=-14, \quad Z=2.74$, $\left.k=87, p_{\text {corr }}=0.0054\right)$. Also, using the contrast social phobia-related words $v s$ neutral words yielded significant associations of amygdala responsiveness with mean methylation (right: $x=24, y=2, z=-22, Z=2.26, k=127$, $p_{\text {corr }}=0.0014$; left: $x=-20, y=-2, z=-24, Z=2.50$, $k=61, p_{\text {corr }}=0.017$ ) and methylation at CpG3 (right: $x=24, y=2, z=-20, Z=2.14, k=65, p_{\text {corr }}=0.0148$; left: $x=-20, y=-2, z=-24, Z=2.22, k=22, p_{\text {corr }}=0.0962$, trend).

\section{DISCUSSION}

In this first multilevel epigenetic study of OXTR methylation in categorical, dimensional, and neuroendocrinological/ neural network intermediate phenotypes of social anxiety, decreased OXTR methylation of exon 3 particularly at CpG3 (Chr3: 8809 437) was significantly associated (1) with the categorical phenotype of SAD, (2) with dimensional traits related to social anxiety (SPS/SIAS scores), (3) with increased cortisol response to the TSST in healthy subjects, and (4) with increased amygdala activation during social phobia-related word processing in patients with SAD. 

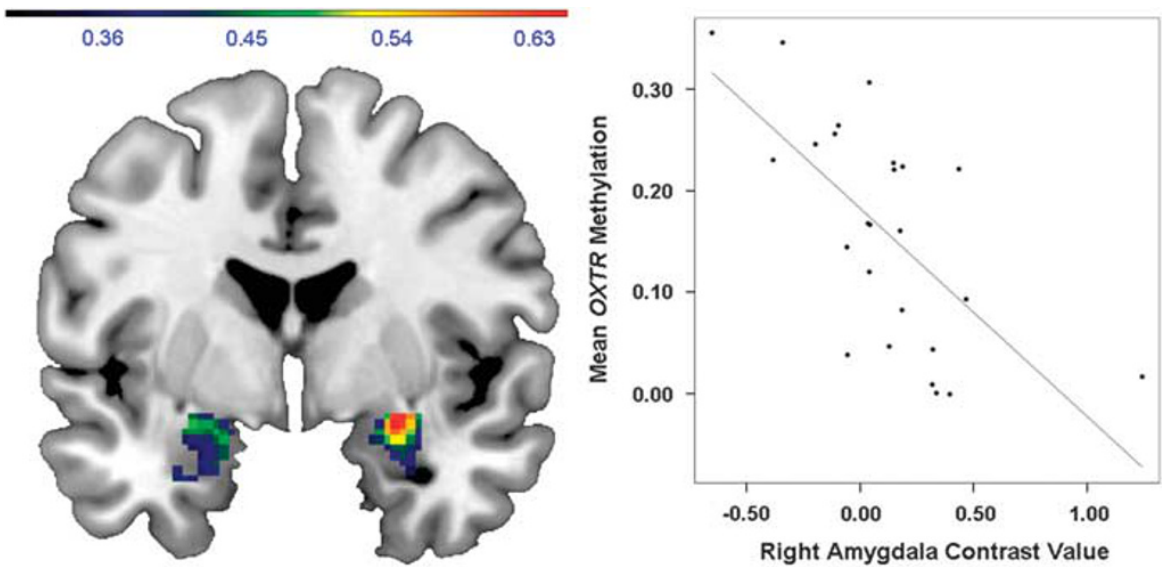

Figure 5 Association of mean OXTR methylation across all 12 CpGs and amygdala responsiveness to social phobia-related words vs negative words in patients with social anxiety disorder $(N=25)$. (Left) Coronal view depicting the negative correlation thresholded at $p<0.05$, $k=38$, yielding a clustercorrected $p<0.05$. Color bar, correlation coefficient $r$. (Right) Scatter plot depicting the correlation of mean OXTR methylation rate across all I2 CpGs and the fMRI contrast value extracted from $x=26, y=-2, z=-14(r=-0.663, p=0.0003)$.

Previously, decreased baseline oxytocin plasma levels were observed to be associated with SAD (Hoge et al, 2012), and intranasal application of oxytocin adjunct to psychotherapy significantly improved self-reported speech performance in SAD patients (Guastella et al, 2009). Assuming decreased OXTR methylation resulting in increased OXTR mRNA expression (see Introduction; Dadds et al, 2014; Gregory et al, 2009; Kusui et al, 2001; Mamrut et al, 2013), the present finding of OXTR hypomethylation being associated with SAD as well as with social phobia-related dimensional measures may reflect a compensatory mechanism leading to an upregulation of oxytocin receptor expression against the background of pathologically low oxytocin levels in SAD and social phobia-related phenotypes. This interpretation is reciprocally analogous to studies showing oxytocin-induced OXTR desensitization as evidenced by downregulated OXTR mRNA (Phaneuf et al, 2000), and is furthermore consistent with significantly decreased OXTR methylation $90 \mathrm{~min}$ after stress exposure (TSST) potentially indicating a compensatory upregulation of the oxytocin system serving to buffer social stress (Unternaehrer et al, 2012; for review see Marazziti et al, 2014).

Accordingly, the present finding of decreased OXTR methylation being associated with an increased cortisol response to the TSST in healthy subjects provides substantial support for the notion of a compensatory mechanism against the background of an impaired physiological stress buffering function because of a lower overall oxytocin tone in certain individuals. These results are in keeping with reports of a negative relationship between endogenous oxytocin and salivary cortisol levels during the TSST (Pierrehumbert et al, 2010) and of intranasal oxytocin administration attenuating the stress-induced cortisol response to the TSST in healthy subjects receiving social support (Heinrichs et al, 2003).

OXTR hypomethylation-potentially reflecting an overall decreased oxytocin tone as discussed above-was furthermore associated with increased amygdala responsiveness to social phobia-related verbal stimuli in patients with SAD. This again is in line with previous reports of oxytocin administration resulting in attenuated amygdala activation in response to socially relevant or fear-conditioned emotional stimuli in healthy subjects as well as in patients with SAD (Domes et al, 2007a; Kirsch et al, 2005; Labuschagne et al, 2010; Petrovic et al, 2008). SAD has frequently been characterized by increased limbic and particularly amygdala hyperresponsiveness to social threat stimuli, in particular social phobia-related words (Schmidt et al, 2010). Accordingly, fMRI studies showed positive associations of amygdala responsiveness and dimensional measures of anxiety and social anxiety (Laeger et al, 2012; Phan et al, 2006). Given the strong expression of OXTR in the amygdala (Bale et al, 2001), one might speculate that OXTR hypomethylation is a mediator of increased vulnerability to and maintenance of SAD by inducing disorder-relevant emotion processing biases based on limbic hyperresponsiveness to signals of rejection, defeat, or humiliation. Hence, the present results further underline the suggested pivotal role of oxytocin in modulating the emotionprocessing neural network implicated in SAD.

The present findings provide converging evidence for an association of OXTR hypomethylation with SAD and social phobia-related traits, suggesting that a decreased oxytocin tone may be involved in the pathophysiology of SAD. However, given the nuanced role of oxytocin in different aspects of social interaction and anxiety traits, its interplay with other neuromodulators, and the complex relation of peripheral and central mechanisms (see Macdonald and Feifel, 2014), the interpretation given above might not fully capture the intricacy of oxytocin system functioning in social cognition and behavior. Notably, several findings challenge the overly simplistic view of oxytocin as an 'anxiolytic' or 'prosocial peptide'. For instance, increased oxytocin plasma levels were associated with social anxiety symptom severity in patients with SAD (Hoge et al, 2008). Moreover, a positive correlation between oxytocin levels and anxiety symptoms was observed regarding experience in close relationships (Marazziti et al, 2006). In addition, oxytocin did not increase approach behavior to threatening stimuli in high social anxiety (Radke et al, 2013). Furthermore, adults with autistic spectrum disorder (ASD) showed increased basal oxytocin levels (Jansen et al, 2006), 
and oxytocin intensified the salience of social stimuli (Domes et al, 2007b; Guastella et al, 2008). Finally, oxytocin was associated with increased amygdala reactivity to scenes depicting social and nonsocial threat in healthy female individuals (Lischke et al, 2012). Thus, the present finding of OXTR hypomethylation-most probably increasing OXTR expression and therefore oxytocin tone-rather than reflecting compensatory upregulation could also be interpreted as an etiological risk factor along the lines of 'when too much of a good thing is bad' as discussed by Young (2013). In sum, the role of OXTR methylation status in social anxiety is still highly speculative warranting thorough investigation in the future, preferably in longitudinal studies in order to determine whether the presently observed OXTR hypomethylation status is a cause or consequence of social anxiety.

Interestingly, although we failed to discern association of OXTR rs53576 genotype with the categorical phenotype of SAD, decreased OXTR methylation was predominant in patients carrying the OXTR rs53576 A allele, and this has previously been reported to constitute a risk allele for lower empathy, optimism, and self-esteem, on one hand, and more concerns about negative perceptions of company as well as higher levels of state loneliness and physiological and dispositional stress reactivity on the other hand (see Kumsta and Heinrichs, 2013). The failure to replicate categorical associations in complex genetic phenotypes such as SAD is in accordance with the notion that direct association between genotype and phenotype might be masked or revealed, respectively, by epigenetic modifications. Allele-specific methylation (ASM) as presently detected has previously been discerned in psychiatric phenotypes (see, eg, Klengel et al, 2013) and might partly account for the 'missing heritability' troubling the field of complex genetics (cf. Meaburn et al, 2010).

The present results should be interpreted in light of some limitations: presently, it was not investigated whether differences in OXTR methylation relate to oxytocin peptide levels or lead to any difference in OXTR expression or brain function, respectively. Although located within a CpG island previously found to influence OXTR expression (see Introduction), the exact functional relevance of the presently investigated CpGs on an mRNA and protein level therefore remains to be elucidated, particularly given their location in exon 3, that is, the first translated exon rather than the promoter region of the gene (cf. Figure 1; Unternaehrer et al, 2012). Furthermore, it is duly noted that DNA methylation patterns in peripheral biomaterial most certainly do not allow for direct conclusions regarding the respective DNA methylation patterns in brain tissue, although Gregory et al (2009) reported high similarity of OXTR methylation patterns in peripheral blood cells and the temporal cortex. Also, several rodent, monkey, and human studies provide support for a certain comparability between DNA methylation in peripheral blood cells/saliva and several brain regions in general (Davies et al, 2012; Provencal et al, 2012; Ursini et al, 2011; for detailed discussion see Domschke et al, 2012). A confounding effect of medication (Uvnas-Moberg et al, 1999) or comorbidity with depression (Parker et al, 2010) is highly unlikely, as all patients but one were not taking any psychoactive medication and no effect of comorbidity with depression on OXTR methylation was found. However, stressful life events not accounted for in our study might have confounded the present results, given dynamic regulation of OXTR methylation after acute psychosocial stress (Unternaehrer et al, 2012). Along these lines, it has to be taken into account that chronic stress or cortisol levels might have confounded the present results, particularly as stress response has not been manipulated pharmacologically in this study. Finally, although we did not detect an effect of sex on overall OXTR methylation or methylation at single $\mathrm{CpGs}$ in the analyzed amplicon, a conclusive interpretation of the present findings regarding the impact of sex is not possible given the heterogeneity of the present samples/subsamples, with the overall sample being heavily weighted for females, whereas the fMRI subsample was exclusively female and the TSST sample had approximately equal proportions of both sexes.

In summary, the present findings for the first time provide converging multilevel evidence for OXTR hypomethylation to be associated with SAD and social phobiarelated traits potentially reflecting a decreased oxytocin tone to be pathogenetically relevant in SAD. Given robust replication of the present results, OXTR methylation patterns as peripheral surrogates of oxytocin tone hopefully will aid in establishing accessible biomarkers of SAD, possibly allowing for indicated preventive interventions as well as more personalized treatment approaches targeting the oxytocin system.

\section{FUNDING AND DISCLOSURE}

The authors declare no conflict of interest. All affiliations mentioned below have no relevance to the work covered in the manuscript: KD has received speaker fees from Pfizer, Lilly, and Bristol-Myers Squibb, she was a consultant for Johnson \& Johnson, and received funding by Astra Zeneca. PZ has received speaker fees from Pfizer, Servier, Lilly, Astra Zeneca, Hexal, and Bristol-Myers Squibb, he is on the advisory board of Pfizer, Merz, and Servier, a consultant for Ironwood Pharmaceuticals, and has received funding from Astra Zeneca and Servier. JH has received speaker's honoraria from Astra-Zeneca. JD has received grant support by Medice. KPL has received speaker fees from Lilly and Astra Zeneca, and grant support by Medice. VA is a member of advisory boards and/or gave presentations for the following companies: Astra-Zeneca, Janssen-Organon, Lilly, Lundbeck, Servier, Pfizer, and Wyeth. He chaired the committee for the "Wyeth Research Award Depression and Anxiety'.

\section{ACKNOWLEDGEMENTS}

The present project was supported by the Deutsche Forschungsgemeinschaft (DFG), SFB-TRR-58, projects C02 (to KD, KPL, and JD), Z02 (to JD and AR) and A05 (to KPL), and FOR 2107, project WP1 (to UD), a grant by the Interdisziplinäres Zentrum für Klinische Forschung (IZKF), University of Muenster (Do3/021/10 to CD and PZ), and the European Community (AGGRESSOTYPE FP7/No. 602805 to $\mathrm{AR}$ and KPL). The contributions of $\mathrm{JH}$ and $\mathrm{CK}$ were supported by the Deutsche Forschungsgemeinschaft (DFG), 
grants HO $1900 / 6-1$ to JH and KI 537/26-1 to CK. AR was supported by the Interdisziplinäres Zentrum für Klinische Forschung (IZKF), University of Wuerzburg (N-221). We gratefully acknowledge the skilful technical and intellectual support by Dr Nicola Tidow, Carola Gagel, and Kathrin Schwarte.

\section{REFERENCES}

Alasaari J-S, Lagus M, Ollila H-M, Toivola A, Kivimaki M, Vahtera J et al (2012). Environmental stress affects DNA methylation of a $\mathrm{CpG}$ rich promoter region of serotonin transporter gene in a nurse cohort. PLoS One 7: e45813.

American Psychiatric Association (2013). Diagnostic and Statistical Manual of Mental Disorders In.American Psychiatric Publishing: Arlington, VA.

Baayen R, Piepenbrock R, Gulikers L (1995). The CELEX lexical database (Release 2). Linguistic Data Consortium, University of Pennsylvania: Pennsylvania, PA.

Bakermans-Kranenburg M-J, van Ijzendoorn M-H (2013). Sniffing around oxytocin: review and meta-analyses of trials in healthy and clinical groups with implications for pharmacotherapy. Transl Psychiatry 3: e258.

Bale TL, Davis AM, Auger AP, Dorsa DM, McCarthy MM (2001). CNS region-specific oxytocin receptor expression: importance in regulation of anxiety and sex behavior. J Neurosci 21: 2546-2552.

Dadds M-R, Moul C, Cauchi A, Dobson-Stone C, Hawes D-J, Brennan J et al (2014). Methylation of the oxytocin receptor gene and oxytocin blood levels in the development of psychopathy. Dev Psychopathol 26: 33-40.

Davies M-N, Volta M, Pidsley R, Lunnon K, Dixit A, Lovestone S et al (2012). Functional annotation of the human brain methylome identifies tissue-specific epigenetic variation across brain and blood. Genome Biol 13: R43.

Domes G, Heinrichs M, Glascher J, Buchel C, Braus D-F, Herpertz S-C (2007a). Oxytocin attenuates amygdala responses to emotional faces regardless of valence. Biol Psychiatry 62: 1187-1190.

Domes G, Heinrichs M, Michel A, Berger C, Herpertz S-C (2007b). Oxytocin improves "mind-reading" in humans. Biol Psychiatry 61: 731-733.

Domschke K, Stevens S, Beck B, Baffa A, Hohoff C, Deckert J et al (2009). Blushing propensity in social anxiety disorder: influence of serotonin transporter gene variation. J Neural Transm 116: 663-666.

Domschke K, Tidow N, Kuithan H, Schwarte K, Klauke B, Ambree O et al (2012). Monoamine oxidase A gene DNA hypomethylation - a risk factor for panic disorder? Int J Neuropsychopharmacol 15: 1217-1228.

Domschke K, Tidow N, Schrempf M, Schwarte K, Klauke B, Reif A et al (2013). Epigenetic signature of panic disorder: a role of glutamate decarboxylase 1 (GAD1) DNA hypomethylation? Prog Neuropsychopharmacol Biol Psychiatry 46: 189-196.

Domschke K, Tidow N, Schwarte K, Deckert J, Lesch KP, Arolt V et al (2014a). Serotonin transporter gene hypomethylation predicts impaired antidepressant treatment response. Int $J$ Neuropsychopharmacol 17: 1167-1176.

Domschke K, Tidow N, Schwarte K, Ziegler C, Lesch KP, Deckert J et al (2014b). Pharmacoepigenetics of depression: no major influence of MAO-A DNA methylation on treatment response. J Neural Transm 122: 99-108.

Faul F, Erdfelder E, Lang AG, Buchne A (2007). G*Power 3: a flexible statistical power analysis program for the social, behavioral, and biomedical sciences. Behav Res Meth 39: 175-191.

Forman S-D, Cohen J-D, Fitzgerald M, Eddy W-F, Mintun M-A, Noll D-C (1995). Improved assessment of significant activation in functional magnetic resonance imaging (fMRI): use of a cluster-size threshold. Magn Reson Med 33: 636-647.

Gregory S-G, Connelly J-J, Towers A-J, Johnson J, Biscocho D, Markunas C-A et al (2009). Genomic and epigenetic evidence for oxytocin receptor deficiency in autism. BMC Med 7: 62-67.

Guastella A-J, Howard A-L, Dadds M-R, Mitchell P, Carson D-S (2009). A randomized controlled trial of intranasal oxytocin as an adjunct to exposure therapy for social anxiety disorder. Psychoneuroendocrinology 34: 917-923.

Guastella A-J, Mitchell P-B, Dadds M-R (2008). Oxytocin increases gaze to the eye region of human faces. Biol Psychiatry 63: 3-5.

Heinrichs M, Baumgartner T, Kirschbaum C, Ehlert U (2003). Social support and oxytocin interact to suppress cortisol and subjective responses to psychosocial stress. Biol Psychiatry 54: 1389-1398.

Hoge E-A, Lawson E-A, Metcalf C-A, Keshaviah A, Zak P-J, Pollack M-H et al (2012). Plasma oxytocin immunoreactive products and response to trust in patients with social anxiety disorder. Depress Anxiety 29: 924-930.

Hoge E-A, Pollack M-H, Kaufman R-E, Zak P-J, Simon N-M (2008). Oxytocin levels in social anxiety disorder. CNS Neurosci Ther 14: $165-170$

Jack A, Connelly J-J, Morris J-P (2012). DNA methylation of the oxytocin receptor gene predicts neural response to ambiguous social stimuli. Front Hum Neurosci 6: 1-7.

Jansen L-M, Gispen-de Wied C-C, Wiegant V-M, Westenberg H-G, Lahuis B-E, van Engeland H (2006). Autonomic and neuroendocrine responses to a psychosocial stressor in adults with autistic spectrum disorder. J Autism Dev Disord 36: 891-899.

Kirsch P, Esslinger C, Chen Q, Mier D, Lis S, Siddhanti S et al (2005). Oxytocin modulates neural circuitry for social cognition and fear in humans. J Neurosci 25: 11489-11493.

Kirschbaum C, Kudielka B-M, Gaab J, Schommer N-C, Hellhammer D-H (1999). Impact of gender, menstrual cycle phase, and oral contraceptives on the activity of the hypothalamuspituitary-adrenal axis. Psychosom Med 61: 154-162.

Kirschbaum C, Pirke K-M, Hellhammer D-H (1993). The 'Trier Social Stress Test'-a tool for investigating psychobiological stress responses in a laboratory setting. Neuropsychobiology 28: $76-81$.

Kirschbaum C, Wüst S, Strasburger C-J (1992). 'Normal' cigarette smoking increases free cortisol in habitual smokers. Life Sci 50: 435-442.

Kissler J, Herbert C, Peyk P, Junghofer M (2007). Buzzwords: early cortical responses to emotional words during reading. Psychol Sci 18: $475-480$.

Klengel T, Mehta D, Anacker C, Rex-Haffner M, Pruessner JC, Pariante CM et al (2013). Allele-specific FKBP5 DNA demethylation mediates gene-childhood trauma interactions. Nat Neurosci 16: 33-41.

Kudielka B-M, Hellhammer D-H, Kirschbaum C (2007). Ten years of research with the trier social stress test-revisited. In: HarmonJones EWinkielman P (eds) Social Neuroscience: Integrating Biological and Psychological Explanations of Social Behavior. Guilford Press: New York, pp 56-83.

Kumsta R, Heinrichs M (2013). Oxytocin, stress and social behavior: neurogenetics of the human oxytocin system. Curr Opin Neurobiol 23: 11-16.

Kumsta R, Hummel E, Chen F-S, Heinrichs M (2013). Epigenetic regulation of the oxytocin receptor gene: implications for behavioral neuroscience. Front Neurosci 7: 1-6.

Kusui C, Kimura T, Ogita K, Nakamura H, Matsumura Y, Koyama M et al (2001). DNA methylation of the human oxytocin receptor gene promoter regulates tissue-specific gene suppression. Biochem Biophys Res Commun 289: 681-686.

Labuschagne I, Phan K-L, Wood A, Angstadt M, Chua P, Heinrichs M et al (2010). Oxytocin attenuates amygdala reactivity to fear in 
generalized social anxiety disorder. Neuropsychopharmacology 35: 2403-2413.

Laeger I, Dobel C, Dannlowski U, Kugel H, Grotegerd D, Kissler J et al (2012). Amygdala responsiveness to emotional words is modulated by subclinical anxiety and depression. Behav Brain Res 233: 508-516.

Laeger I, Dobel C, Radenz B, Kugel H, Keuper K, Eden A et al (2014a). Of 'disgrace' and 'pain' - corticolimbic interaction patterns for disorder-relevant and emotional words in social phobia. PloS One 9: e109949.

Laeger I, Keuper K, Heitmann C, Kugel H, Dobel C, Eden A et al (2014b). Have we met before? Neural correlates of emotional learning in women with social phobia. J Psychiatry Neurosci 39: E14-E23.

Lewin J, Schmitt A-O, Adorjan P, Hildmann T, Piepenbrock C (2004). Quantitative DNA methylation analysis based on fourdye trace data from direct sequencing of PCR amplificates. Bioinformatics 20: 3005-3012.

Lischke A, Gamer M, Berger C, Grossmann A, Hauenstein K, Heinrichs $\mathrm{M}$ et al (2012). Oxytocin increases amygdala reactivity to threatening scenes in females. Psychoneuroendocrinology 37: 1431-1438.

Macdonald K, Feifel D (2014). Oxytocins role in anxiety: a critical appraisal. Brain Res 1580: 22-56.

Mamrut S, Harony H, Sood R, Shahar-Gold H, Gainer H, Shi YJ et al (2013). DNA methylation of specific CpG sites in the promoter region regulates the transcription of the mouse oxytocin receptor. PLoS One 8: e56869.

Marazziti D, Abelli M, Baroni S, Carpita B, Ramacciotti C-E, Dell'Osso L (2014). Neurobiological correlates of social anxiety disorder: an update. CNS Spectrums 26: 1-12.

Marazziti D, Dell'Osso B, Baroni S, Mungai F, Catena M, Rucci P et al (2006). A relationship between oxytocin and anxiety of romantic attachment. Clin Pract Epidemiol Ment Health 2: 2-28.

Meaburn EL, Schalkwyk LC, Mill J (2010). Allele-specific methylation in the human genome: implications for genetic studies of complex disease. Epigenetics 5: 578-582.

Meyer-Lindenberg A, Domes G, Kirsch P, Heinrichs M (2011). Oxytocin and vasopressin in the human brain: social neuropeptides for translational medicine. Nat Rev Neurosci 12: 524-538.

Miller R, Plessow F, Kirschbaum C, Stadler T (2013). Classification criteria for distinguishing cortisol responders from nonresponders to psychosocial stress: evaluation of salivary cortisol pulse detection in panel designs. Psychosom Med 75: 832-840.

Ohayon MM, Schatzberg AF (2010). Social phobia and depression: prevalence and comorbidity. J Psychosom Res 68: 235-243.

Parker K-J, Kenna H-A, Zeitzer J-M, Keller J, Blasey C-M, Amico J-A et al (2010). Preliminary evidence that plasma oxytocin levels are elevated in major depression. Psychiatry Res 178: 359-362.

Petrovic P, Kalisch R, Singer T, Dolan R-J (2008). Oxytocin attenuates affective evaluations of conditioned faces and amygdala activity. J Neurosci 28: 6607-6615.

Phan K-L, Fitzgerald D-A, Nathan P-J, Tancer M-E (2006). Association between amygdala hyperactivity to harsh faces and severity of social anxiety in generalized social phobia. Biol Psychiatry 59: 424-429.

Phaneuf S, Rodriguez L-B, TambyRaja R-L, MacKenzie I-Z, Lopez B-A (2000). Loss of myometrial oxytocin receptors during oxytocin-induced and oxytocin-augmented labour. J Reprod Fertil 120: 91-97.

Pierrehumbert B, Torrisi R, Laufer D, Halfon O, Ansermet F \& Beck Popovic M (2010). Oxytocin response to an experimental psychosocial challenge in adults exposed to traumatic experiences during childhood or adolescence. Neuroscience 166: 168-177.
Provencal N, Suderman M-J, Guillemin C, Massart R, Ruggiero A, Wang $\mathrm{D}$ et al (2012). The signature of maternal rearing in the methylome in rhesus macaque prefrontal cortex and $\mathrm{T}$ cells. J Neurosci 32: 15626-15642.

Radke S, Roelofs K, de Bruijn E-R (2013). Acting on anger: social anxiety modulates approach-avoidance tendencies after oxytocin administration. Psychol Sci 24: 1573-1578.

Rodrigues S-M, Saslow L-R, Garcia N, John O-P, Keltner D (2009). Oxytocin receptor genetic variation relates to empathy and stress reactivity in humans. Proc Natl Acad Sci USA 106: 21437-21441.

Saphire-Bernstein S, Way B-M, Kim H-S, Sherman D-K, Taylor S-E (2011). Oxytocin receptor gene (OXTR) is related to psychological resources. Proc Natl Acad Sci USA 108: 15118-15122.

Schmidt S, Mohr A, Miltner W-H-R, Straube T (2010). Taskdependent neural correlates of the processing of verbal threatrelated stimuli in social phobia. Biol Psychology 84: 304-312.

Stangier U, Heidenreich T, Berardi A, Golbs U, Hoyer J (1999). Die Erfassung Sozialer Phobie durch Social Interaction Anxiety Scale (SIAS) und die Social Phobia Scale (SPS). Zeitschrift für Klinische Psychologie 28: 28-36.

Stein M-B, Stein D-J (2008). Social anxiety disorder. Lancet 371: $1115-1125$.

Stein MB, Tancer ME, Gelernter CS, Vittone BJ, Uhde TW (1990). Major depression in patients with social phobia. Am J Psychiatry 147: 637-639.

Stevens S, Gerlach A-L, Rist F (2008). Effects of alcohol on ratings of emotional facial expressions in social phobics. J Anxiety Disord 22: 940-948.

Tadic A, Müller-Engling L, Schlicht K-F, Kotsiari A, Dreimuller N, Kleimann A et al (2013). Methylation of the promoter of brainderived neurotrophic factor exon IV and antidepressant response in major depression. Mol Psychiatry 19: 281-283.

Tzourio-Mazoyer N, Landeau B, Papathanassiou D, Crivello F, Etard O, Delcroix N et al (2002). Automated anatomical labeling of activations in SPM using a macroscopic anatomical parcellation of the MNI MRI single-subject brain. NeuroImage 15: 273-289.

Unternaehrer E, Luers P, Mill J, Dempster E, Meyer A-H, Staehli S et al (2012). Dynamic changes in DNA methylation of stressassociated genes (OXTR, BDNF ) after acute psychosocial stress. Transl Psychiatry 2: e150.

Ursini G, Bollati V, Fazio L, Porcelli A, Iacovelli L, Catalani A et al (2011). Stress-related methylation of the catechol-O-methyltransferase Val 158 allele predicts human prefrontal cognition and activity. J Neurosci 31: 6692-6698.

Uvnas-Moberg K, Bjokstrand E, Hillegaart V, Ahlenius S (1999). Oxytocin as a possible mediator of SSRI-induced antidepressant effects. Psychopharmacology (Berl) 142: 95-101.

Wittchen H-U (1997). SKID-I: Strukturiertes klinisches Interview für DSM-IV, Achse I: Psychische Störungen. Hogrefe: Goettingen.

Wittchen H-U, Pfister H (1997). DIA-X-Interviews: Manual für Screening-Verfahren und Interview; Interviewheft Längsschnittuntersuchung (DIA-X-Lifetime); Ergänzungsheft (DIA-X-Lifetime); Interviewheft Querschnittsuntersuchung (DIA-X-Monate); Ergänzungsheft (DIA-X-Monate); PC-Programm zur Durchführung des Interviews (Längs-und Querschnittsuntersuchung); Auswertungsprogramm. Swets and Zeitlinger: Frankfurt.

Wu S, Jia M, Ruan Y, Liu J, Guo Y, Shuang M et al (2005). Positive association of the oxytocin receptor gene (OXTR) with autism in the Chinese Han population. Biol Psychiatry 58: 74-77.

Young LJ (2013). When too much of a good thing is bad: chronic oxytocin, development, and social impairments. Biol Psychiatry 74: $160-161$.

Supplementary Information accompanies the paper on the Neuropsychopharmacology website (http://www.nature.com/npp) 